\title{
SELF CONSISTENT MONTE CARLO METHOD TO STUDY CSR EFFECTS IN BUNCH COMPRESSORS *
}

\author{
G. Bassi ${ }^{\dagger}$, Department of Physics, University of Liverpool, Liverpool, \\ J. A. Ellison, K. Heinemann, Department of Mathematics, UNM, Albuquerque, \\ R. Warnock, SLAC, Menlo Park, California.
}

\section{INTRODUCTION}

In this paper we report on the results of a self-consistent calculation of CSR effects on a particle bunch moving through the benchmark Zeuthen bunch compressors. The theoretical framework is based on a 4D Vlasov-Maxwell approach including shielding from the vacuum chamber. We calculate the fields in the lab frame, where time is the independent variable, and evolve the phase space density/points in the beam frame, where arc length, $s$, along a reference orbit, is the independent variable. Some details are given in [2], where we also discuss three approaches, the unperturbed source model (UPS), the self constistent Monte Carlo (SCMC) method and the method of local characteristics.

Results for the UPS have been presented for $5 \mathrm{GeV}$ before [3], here we compare them with our new results from the SCMC and study the $500 \mathrm{MeV}$ case. Our work using the method of characteristics is in progress. The SCMC algorithm begins by randomly generating an initial ensemble of beam frame phase space points according to a given initial phase space density. The algorithm then reduces to laying out one arc length step. Assume that at arc length $s$ we know the location of the phase space points and the history of the source prior to $s$. We then i) create a smooth representation of the lab frame charge and current densities, $\rho_{L}$ and $\mathbf{J}_{L}$, ii) calculate the fields at $s$ from the history of $\rho_{L}$ and $\mathbf{J}_{L}$, and iii) move the beam frame phase space points according to the beam frame equations of motion. This is then iterated.

The UPS calculation is similar except the fields are calculated from a function of s computed a priori from the beam frame equations of motion without the self-fields. The phase space points are then evolved according to the equations of motion with these "unperturbed" fields. In the UPS we use a Gaussian initial density which evolves under the linear beam frame equations as a Gaussian. This gives us an analytic formula for the source, which significantly speeds up the field calculation.

It turns out that the evolution of the unperturbed charge density for an initial Gaussian gives a reasonable estimate of the support of the self-consistently calculated charge density in our study so far. This allows us to follow the phase space points in a fixed grid region defined by the

\footnotetext{
* Work supported by DOE grants DE-G02-99ER41104 and DE-AC0276SF00515

† g.bassi@dl.ac.uk
}

mean center of the Gaussian and an orthonormal transformation which takes the Gaussian ellipses into circles. We put the $5 \sigma$ circle into the square $[-1,1]^{2}$ and take this as our basic region for the calculation. Thus at $s$ we have the spatial position of the particles scattered in this square. We then construct a smooth spatial density using a 2D Fourier expansion on the square, calculating the Fourier coefficients from the scattered data, as a Monte Carlo integration. This is a common technique in statistical estimation [4]. This (analytical) density on the square is then used to calculate the source for the field calculation on grid points. Typically we use $32 \times 32$ grid points and $16 \times 16$ Fourier coefficients. The fields are calculated at the grid points and the scattered phase space points are moved by interpolating the fields.

\section{NUMERICAL STUDIES}

In [3] we presented numerical results in the UPS for the benchmark $5 \mathrm{GeV}$ bunch compressor studied in [1]. We gave a complete description of moments and reduced densities with an emphasis on the effects of shielding and the transverse force $F_{x}[2,(16)]$.

In this contribution we show self-consistent results for the benchmark $5 \mathrm{GeV}$ and $500 \mathrm{MeV}$ bunch compressors in the free space case without shielding. The chicane we study has 4 magnets of length $0.5 \mathrm{~m}$. The left corners of the magnets are at $0 \mathrm{~m}, 5.5 \mathrm{~m}, 7 \mathrm{~m}$ and $12.5 \mathrm{~m}$. The total length of the chicane is $15 \mathrm{~m}$. The initial phase space density is Gaussian. In Fig. 1 and in the left frame of Fig. 2 we compare the self-consistent results with the UPS results. We see that the agreement is very good indicating that the UPS is a good approximation at $5 \mathrm{GeV}$. Notice that self-consistently the CSR has a bigger effect than in the UPS. In the right frame of Fig. 2 we plot the charge density at $s=15 \mathrm{~m}$ (end of the chicane)- its support on the normalized grid $[-1,1]^{2}$ is not significantly different from the support of the unperturbed density. The charge density is well represented with $12 \times 12$ Fourier coefficients and $10^{6}$ particles.

In Fig. 3, Fig. 4 and Fig. 5 (here we use $20 \times 20$ Fourier coefficients and $10^{6}$ particles) we show results for the benchmark $500 \mathrm{MeV}$ Gaussian case. We compare a complete simulation in the UPS vs a preliminary simulation (up to $12 \mathrm{~m}$ ) in the SCMC. We are currently studying the self-consistent dynamics in the 4th magnet. CSR here is a stronger perturbation: the change in the energy moments and $x$-emittance due to CSR has increased by a 

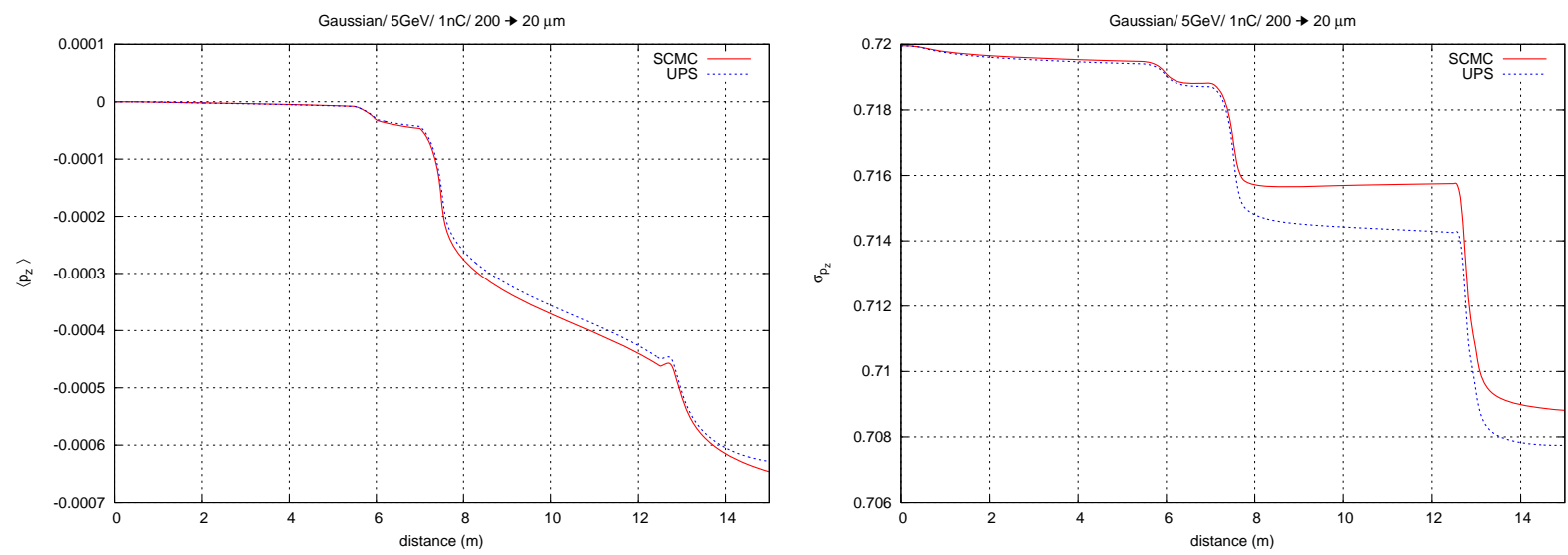

Figure 1: Comparison between SCMC and UPS results at $5 \mathrm{GeV}$. Left: mean energy loss $\left\langle p_{z}\right\rangle$. Right: standard deviation of relative energy deviation $\sigma_{p_{z}}$. In both cases the self-consistent results with SCMC are slightly bigger than the UPS results.
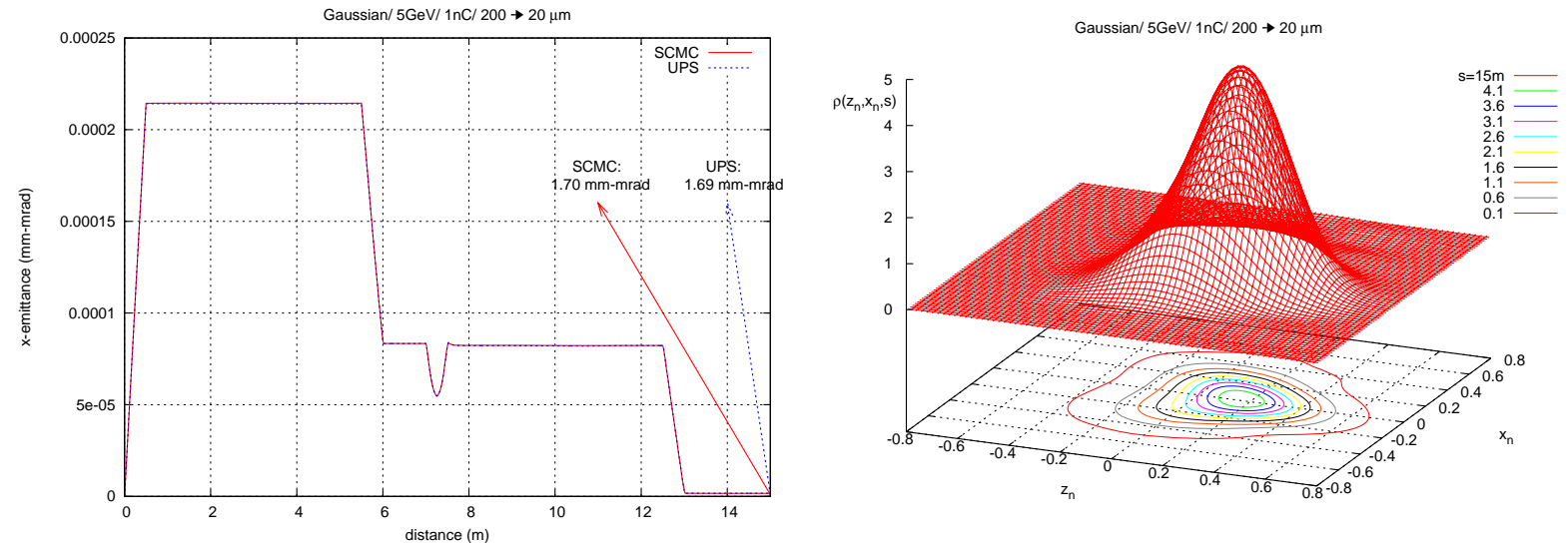

Figure 2: Left: comparison between SCMC and UPS at $5 \mathrm{GeV}$ : $x$-emittance. Here the $x$-emittance calculated with SCMC is also slightly bigger than the UPS results. Right: SCMC spatial density at $s=15 \mathrm{~m}$ (end of the chicane) at $5 \mathrm{GeV}$. Notice the SCMC support is close to the support of the UPS spatial density.
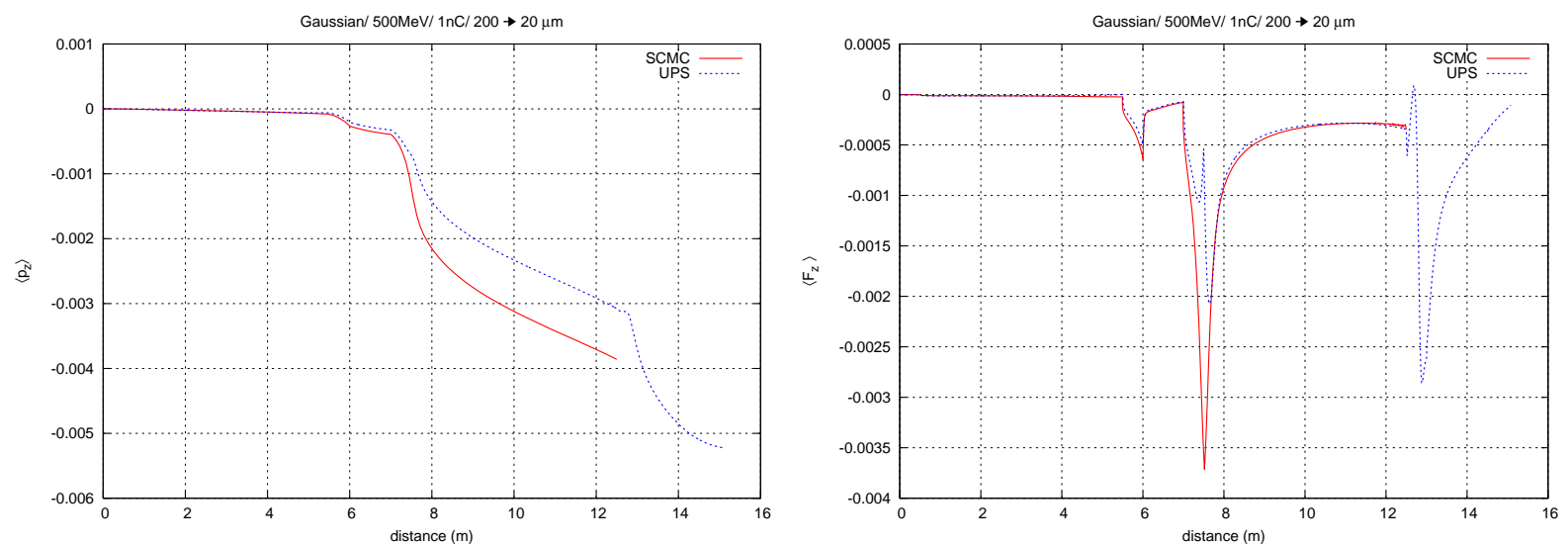

Figure 3: Comparison between SCMC and UPS results at $500 \mathrm{MeV}$. Left: mean energy loss $\left\langle p_{z}\right\rangle$. Right: Mean power $\left\langle F_{z}\right\rangle$. Here CSR effects are 10 times stronger than at $5 \mathrm{GeV}$. Notice the agreement is not as good as at $5 \mathrm{GeV}$. 

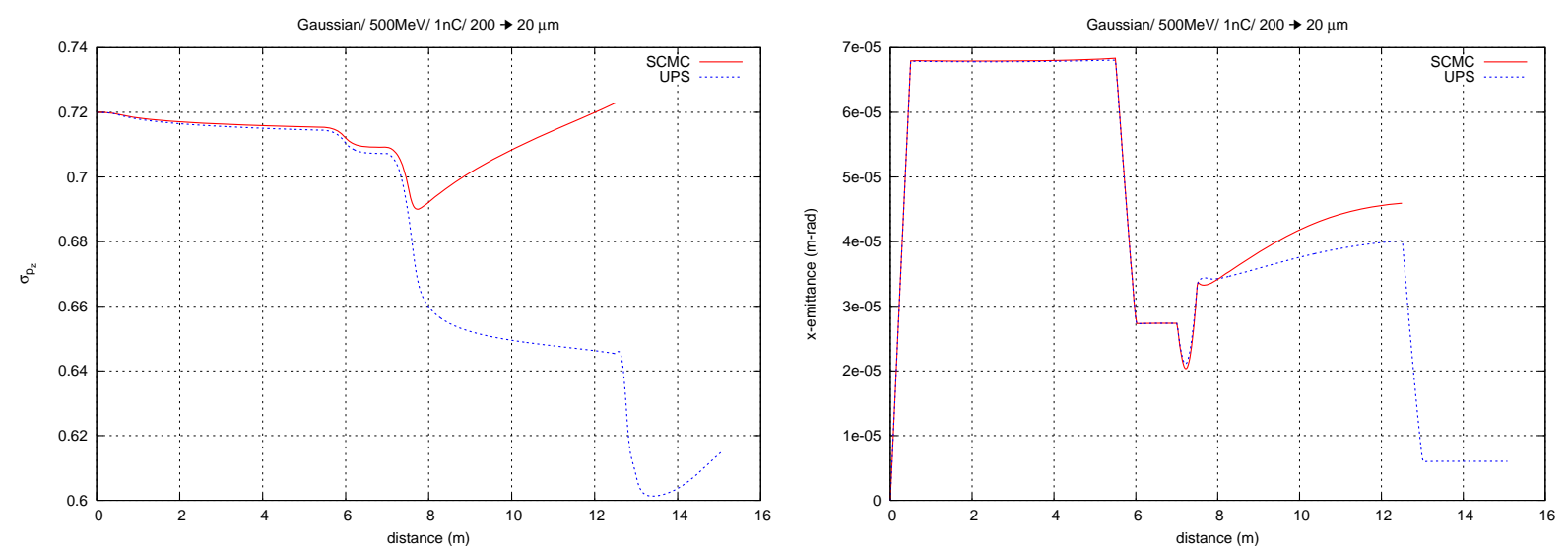

Figure 4: Comparison between SCMC and UPS results at $500 \mathrm{MeV}$. Left: standard deviation of relative energy deviation $\sigma_{p_{z}}$. Right: $x$-emittance. Here CSR effects are similar to the effect on the mean energy loss and mean power.
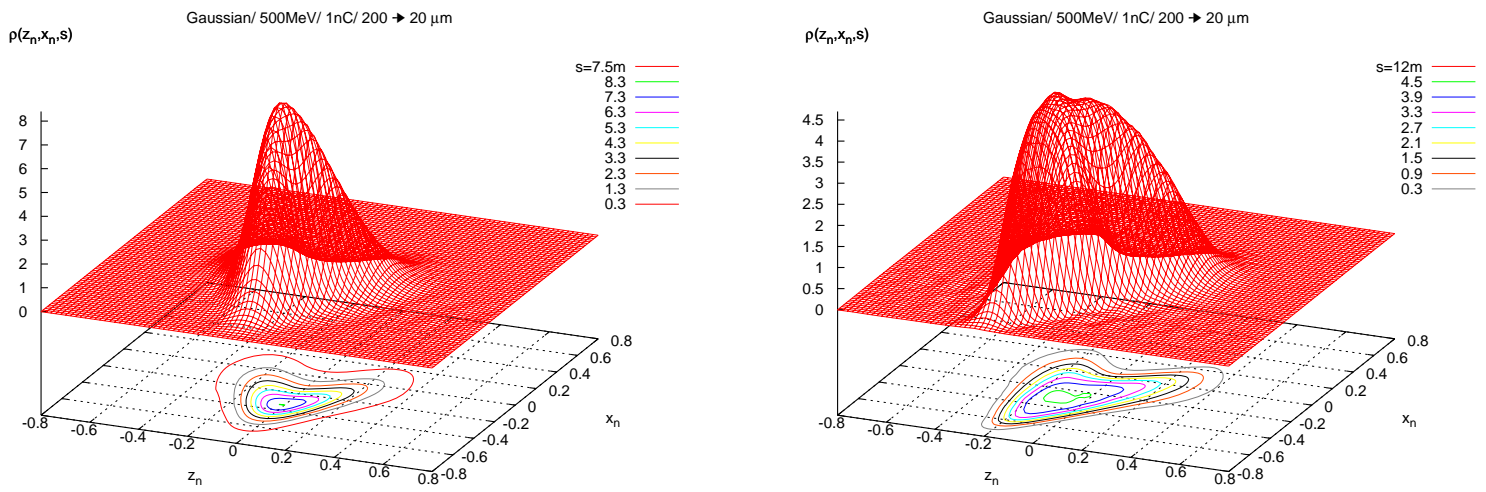

Figure 5: SCMC charge density at $500 \mathrm{MeV}$ : Left: $s=7.5 \mathrm{~m}$ (entrance 3rd magnet). Right: $s=12 \mathrm{~m}(0.5 \mathrm{~m}$ before the entrance 4 th magnet). Notice the charge density has a different support than the unperturbed charge density and has more structure.

factor of 10 with respect to the $5 \mathrm{GeV}$ case. In particular, notice that the final $x$-emittance is 6 times the unperturbed $x$-emittance $\left(10^{-6} \mathrm{~m}\right.$-rad $)$. Moreover the support of the perturbed charge density differs from the unperturbed one and the density has more structure. A self consistent study therefore is more challenging at $500 \mathrm{MeV}$. More structure means more Fourier coefficients are needed. A crucial issue is the choice of the number of Fourier coefficients to accurately represent the charge density. In Fig. 5 we show preliminary self consistent results at $s=7 \mathrm{~m}$ and $s=12 \mathrm{~m}$ along the chicane. Our strategy to follow the self consistent evolution of the system is to progressively increase the number of Fourier coefficients.

Our future plan consists in completing the self consistent study of the $500 \mathrm{MeV}$ Gaussian case and the study of different initial distributions (longitudinal parabolic distribution and nonlinear chirp) both at $5 \mathrm{GeV}$ and $500 \mathrm{MeV}$. Shielding effects will be included.

We will focus then on the development of the method of local characteristics. As pointed out in [3], scatter plots of the interaction picture variable $w=p_{z 0}-u z_{0}$ vs $z_{0}$ indicate the need of a moving grid for strongly correlated systems, a non trivial task.

\section{Acknowledgments}

Our simulations have been done at the Center for High Performance Computing at UNM and on NERSC at LBNL. We gratefully acknowledge T. Thomas and R. Heimbach at UNM and R. Ryne at LBNL for giving us an account on NERSC.

\section{REFERENCES}

[1] ICFA Beam Dynamics Mini-Workshop on CSR, BerlinZeuthen, 2002, see http//www.desy.de/csr; L. Giannessi and M. Quattromini, PRSTAB 6, 120101 (2003).

[2] J. A. Ellison, G. Bassi, K. Heinemann, M. Venturini and R. Warnock. These Proceedings.

[3] G. Bassi, J. A. Ellison, K. Heinemann, Proceedings of EPAC2006, Edinburgh, Scotland.

[4] S. Efromovich, 'Nonparametric Curve Estimation: Methods, Theory, and Applications'. Springer 1999. 